\title{
Canine small clear cell/T-zone lymphoma: clinical presentation and outcome in a retrospective case series
}

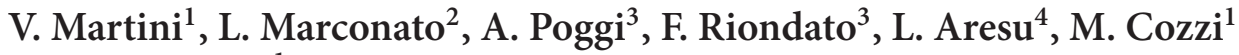 \\ and S. Comazzi ${ }^{1}$ \\ ${ }^{1}$ Department of Veterinary Sciences and Public Health, University of Milan, Milan, Italy \\ ${ }^{2}$ Centro Oncologico Veterinario, Bologna, Italy \\ ${ }^{3}$ Department of Veterinary Science, University of Turin, Turin, Italy \\ ${ }^{4}$ Department of Comparative Biomedicine and Food Science, University of Padua, Padua, Italy
}

\section{Keywords}

CD45-negative, clinical presentation, lymphoma, outcome, small clear cell, T-zone
Correspondence address: V. Martini

Department of Veterinary Sciences and Public Health Faculty of Veterinary Medicine University of Milan Via Celoria 10, 20133 Milan Italy

e-mail:

valeria.martini@unimi.it

\begin{abstract}
Published studies, taken together, suggest the existence of a single canine lymphoma entity, with a small clear cell appearance by cytological evaluation, a histopathological T-zone pattern and an aberrant CD45-negative T-cell phenotype, mostly characterized by long-term survival. We describe clinical presentation and outcome in a retrospective case series of canine small clear cell/T-zone lymphoma. Despite the reported predisposition of Golden retriever, this breed was not represented in our case series. Most dogs presented with stage $V$ disease, whereas only few had clinical signs or peripheral cytopenias. Blood was almost always more infiltrated than bone marrow. Median survival confirmed the favourable prognosis described in literature, but a few dogs died within a short time. Also, a subgroup of dogs developed second malignancies, eventually leading to death. We did not investigate possible prognostic factors because of the wide variety in treatments, and further studies are needed to identify high-risk animals.
\end{abstract}

\section{Introduction}

Canine lymphoma is a heterogeneous disease, made up of several distinct subtypes. Many classification systems have been proposed over the years, focusing on different characteristics, including cytological appearance, phenotype and histopathological pattern, thereby reducing the ability to compare results from different studies, because of the varied inclusion criteria and adopted classifications schemes.

In 2010, a new lymphoma classification proposal was published and the authors attempted to merge several morphological features, such as cytology, histopathology and phenotype into one single system. ${ }^{1}$ Interestingly, this study highlighted that lymphomas with cytological small clear cell appearance may be considered suggestive, although not conclusive, of T-cell phenotype and representative of T-zone lymphoma (TZL). More recently, our research group highlighted a high prevalence of phenotypic aberrancies in canine small clear cell lymphoma, mostly a lack of CD45 expression, which accounted for $>95 \%$ of the cases. ${ }^{2}$ At the same time, another study demonstrated that $100 \%$ of canine CD45-negative T-cell neoplasia were histologically defined as TZL. ${ }^{3}$ Taken together, these three studies suggest that lymphomas with a small clear cell appearance by cytology, a nodular pattern of expansion from cells derived from the paracortex by histology and a negative staining for CD45 by flow cytometry (FC), frequently associated with an aberrant CD21 expression, represent a single entity.

To date, the clinical presentation and characteristics of dogs with this lymphoma subtype have only 
Table 1. Antibodies used for flow cytometric immunophenotyping of lymph node from 51 dogs with CD45-negative small clear cells/T-zone lymphoma

\begin{tabular}{llll}
\hline Target molecule & Antibody clone & & \multicolumn{1}{c}{ Source } \\
\hline CD45 & YKIX716.13 & Serotec, Oxford, UK & Specificity \\
CD44 & IM7 & BD Pharmingen, San Diego, CA, USA & All leukocytes \\
CD18 & CA1.4E9 & Serotec & All leukocytes \\
CD3 & CA17.2A12 & Serotec & T-cells \\
CD5 & YKIX322.3 & Serotec & T-cells \\
CD4 & YKIX302.9 & Serotec & T-helper cells and neutrophils \\
CD8 & YCATE55.9 & Serotec & T-cytotoxic cells \\
CD21 & CA2.1D6 & Serotec & Mature B-cells \\
\hline
\end{tabular}

been summarily described. ${ }^{3,4}$ Because of its indolent nature, a long survival is to be expected..$^{3-5}$ However, one study reported a median survival of only 55 days for dogs with indolent T-cell lymphomas and advanced stage, including small lymphocytic lymphomas and TZL. ${ }^{6}$ It may be possible that a subgroup of TZL harbours a poor prognosis. The aim of this study was to describe the clinical presentation and outcome in a retrospective series of canine CD45-negative small clear cell/TZL.

\section{Materials and methods}

The FC databases of the authors' institutions were interrogated from January 2009 to December 2014, and the CD45-negative, small (FSC-height $<400$ ) T-cell lymphoma cases were selected. Additional inclusion criteria were a cytological diagnosis of small clear cell lymphoma and when available a confirmed histopathological diagnosis of TZL. ${ }^{7,8}$

Flow cytometric immunophenotyping on lymph node (LN) aspirates collected into RPMI (Sigma Aldrich, St Louis, MO, USA) was performed as previously described, ${ }^{9}$ using a multicolour approach including antibodies against CD45, CD44, CD18, CD3, CD5, CD21, CD4 and CD8 (Table 1). If available, peripheral blood $(\mathrm{PB})$ and/or bone marrow (BM) samples collected into ethylenediaminetetraacetic acid (EDTA) tubes were analysed with an automated haemocytometer (Sysmex XT-2000iV; Sysmex, Kobe, Japan) and examined via $\mathrm{FC}$ to quantify infiltration. BM samples were considered eligible for FC only if purity was $\geq 80 \%$ according to the following formula: $[1-(B M$ erythrocytes $/ \mathrm{PB}$ erythrocytes $) \times(\mathrm{PB}$ leukocytes $/ \mathrm{BM}$ leukocytes) $] \times 100 \% .{ }^{10}$ Samples were acquired either with a FACScalibur or with a BD Accuri C6 (Becton Dickinson, San Josè, CA, USA) and analysed using the specific software CellQuest Pro or CFlow Plus (Becton Dickinson).

$\mathrm{PB}$ and $\mathrm{BM}$ samples were considered infiltrated if a distinct CD45-negative population with similar phenotype shown in the LN was detected. Infiltration was then quantified as the percentage of neoplastic cells out of the total events acquired, after exclusion of debris and platelets based on morphological scattergrams. The neoplastic phenotype, $\mathrm{PB}$ and $\mathrm{BM}$ infiltration degree were recorded for each case.

Clinical data were obtained from the medical records and by phone calls to the referring veterinarians. Background information collected for each dog included signalment, presence or absence of clinical symptoms, complete blood count (CBC) at diagnosis, treatment (if any), date and cause of death. Haematological abnormalities were defined as values out of the laboratory reference interval (RI).

Clinical stage was based on the World Health Organization (WHO) criteria for canine lymphoma; however, splenic, liver, $\mathrm{PB}$ and BM aspirates were not routinely performed and therefore it was not possible to definitively differentiate stage III from stage IV or V disease in some cases.

Median survival time was calculated via SPSS v17.0 for Windows. Survival time was defined as time between diagnosis and death. Dogs that died or were euthanized because of lymphoma (including deterioration of clinical conditions without any other identifiable cause) were recorded as events; dogs lost to follow up, dead for unrelated causes or still alive at data analysis closure 
were censored. Because of the wide range of treatment protocols, prognostic factors were not investigated.

\section{Results}

\section{Clinical presentation}

Fifty-one dogs met the inclusion criteria. Cytology confirmed a small clear cell appearance in all cases, whereas histopathology confirmed TZL in the eight cases also undergoing lymphadenectomy. Breed was reported for 43 dogs, including 33 (76.7\%) pure breed dogs and 10 (23.3\%) mixed-breed dogs. The most represented breeds were Boxer $(n=5)$, English bulldog $(n=3)$, Labrador retriever $(n=3)$ and Shih tzu $(n=3)$. Sex was reported for 47 dogs. Among these, 23 (48.9\%) were male (2 neutered) and 24 (51.1\%) were female (12 spayed). Age was reported for 44 dogs. Overall mean age was $9.9 \pm 2.3$ years (median 10 years; range, $5-14$ years). In particular, 18 (40.9\%) dogs were $<10$ years old and $26(59.1 \%)$ were $\geq 10$ years old.

CBC data were available for 44 dogs. Four (9.1\%) dogs had a mild anaemia, $3(7.3 \%)$ had thrombocytopenia, 2 (4.5\%) had leukopenia and 17 (38.6\%) had leukocytosis, 4 (9.1\%) had neutropenia and $8(18.2 \%)$ had mature neutrophilia, 2 (4.5\%) had lymphopenia and 28 (63.6\%) had lymphocytosis. Overall mean white blood cell (WBC) count was $17.59 \pm 10.52 \times 10^{3} / \mu \mathrm{L}$ (median $14.39 \times 10^{3} / \mu \mathrm{L}$; range, $\left.0.8-45.72 \times 10^{3} / \mu \mathrm{L}\right)$; overall mean neutrophil count was $8.24 \pm 5.44 \times 10^{3} / \mu \mathrm{L}$ (median $6.73 \times 10^{3} / \mu \mathrm{L} ; \quad$ range, $\left.0.59-27.2 \times 10^{3} / \mu \mathrm{L}\right)$; overall mean lymphocyte count was $8.31 \pm 6.61 \times 10^{3} / \mu \mathrm{L}$ (median $6.95 \times 10^{3} / \mu \mathrm{L}$; range, $\left.0.12-30.76 \times 10^{3} / \mu \mathrm{L}\right)$.

In $20(39.2 \%)$ cases, neoplastic cells were CD8+, in $17(33.3 \%)$ cases cells were CD4-CD8 double negative, in $8(15.7 \%)$ cases cells were CD4+, in 4 (7.8\%) cases cells were CD4-CD8 double positive and in $2(3.9 \%)$ cases two distinct CD45-negative populations were identifiable, staining positive for CD4 and CD8, respectively. Forty-two (82.4\%) cases stained positive for CD21.

Stage was reported for 43 dogs; however, staging procedures largely varied among veterinarians and were not standardized. One (2.3\%) dog had stage I disease, 1 (2.3\%) dog had stage III disease, 1 (2.3\%) dog had stage IV disease and 40 (93\%) dogs were classified as stage $\mathrm{V}$ because of PB and/or BM flow cytometric infiltration; in addition, skin and lungs were presumed to be involved in one case each. The dog with skin involvement presented multifocal itchy alopecia of abdomen, neck and pinnae; histopathological examination of cutaneous biopsies from the neck and the abdomen revealed a diffuse subepidermal infiltration by small lymphocytes. Lung involvement was diagnosed based on thoracic radiographs, that revealed a generalized multifocal interstitial structured pulmonary pattern, and cytological examination of a percutaneous fine needle aspiration, that was suggestive of round cell tumour, whereas bronchoalveolar lavage was negative for lymphomatous cells.

Substage was reported for 22 dogs. Among these, $18(81.8 \%)$ were asymptomatic (substage a), whereas $4(18.2 \%)$ had clinical signs (substage b), mainly dyspnoea because of enlarged submandibular LNs. PB and BM samples were analysed via FC in 40 and 12 dogs, respectively, and all of them proved to be infiltrated. Overall mean PB infiltration was $34.6 \pm 17.53 \%$ (median $35.07 \%$; range, $0.93-64.6 \%)$. Overall mean BM infiltration was $6.85 \pm 7.88 \%$ (median $2.15 \%$; range, $0.5-24.1 \%$ ). In the 12 cases that had BM analysed, mean $\mathrm{PB}$ infiltration was $24.64 \pm 14.41 \%$ (median $27.1 \%$; range, $1.8-41.65 \%)$; in particular, $\mathrm{PB}$ infiltration was higher than $\mathrm{BM}$ in all cases but one. Flow cytometric scattergrams of LN, PB and BM from one representative case are shown in Fig. 1. Clinical features are listed in Table 2.

\section{Outcome}

Follow-up data were available for 26 dogs. Four dogs received no therapy after diagnosis, 3 received corticosteroids alone and the remaining 19 dogs were treated with different protocols, including dose-intense $(n=12)$ and metronomic $(n=7)$ chemotherapy.

Overall median survival was 760 days (range, 15-1150 days). Eight of the 26 dogs died of lymphoma during the study period, with a median survival of 180 days (range, 15-760 days); among them, 3 dogs (37.5\%) died within 3 months from the diagnosis, and 5 (62.5\%) survived longer than 6 

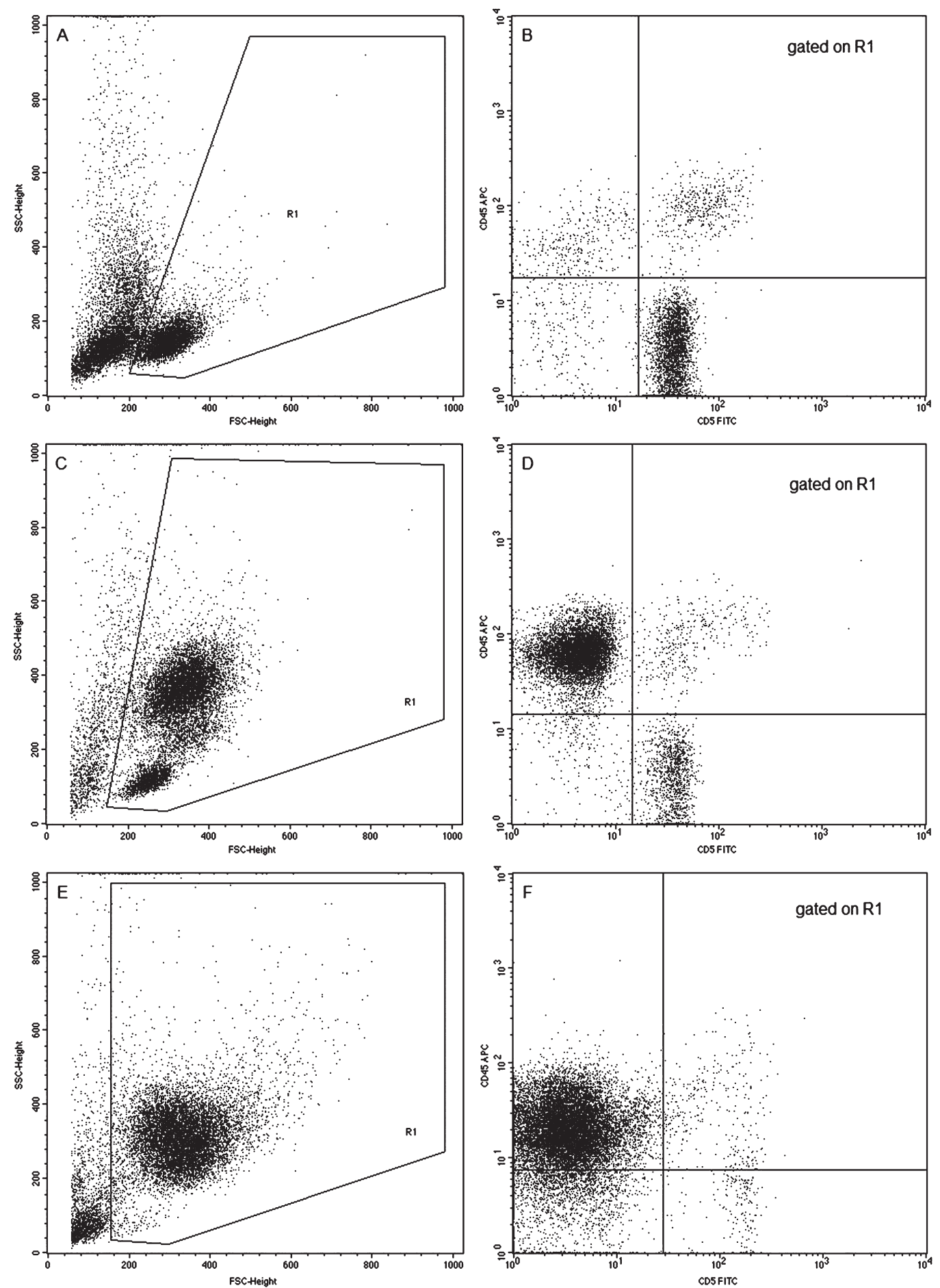

Figure 1. Flow cytometric scattergrams representing lymph node aspirate (A and B), peripheral blood (C and D) and bone marrow (E and F) from a dog with small clear cell/T-zone lymphoma (Table 2, number 7). Events were displayed at first based on morphological properties (A, C and E) and a gate (R1) was set to exclude platelets and debris. R1 cells were then displayed based on CD5-fitc and CD45-apc fluorescence. A distinct CD45-negative and CD5-positive population was identified in all samples (B, D, F, lower right quadrant), accounting for 98.6, 12.2 and $0.95 \%$ of all cells in the lymph node, peripheral blood and bone marrow, respectively. 


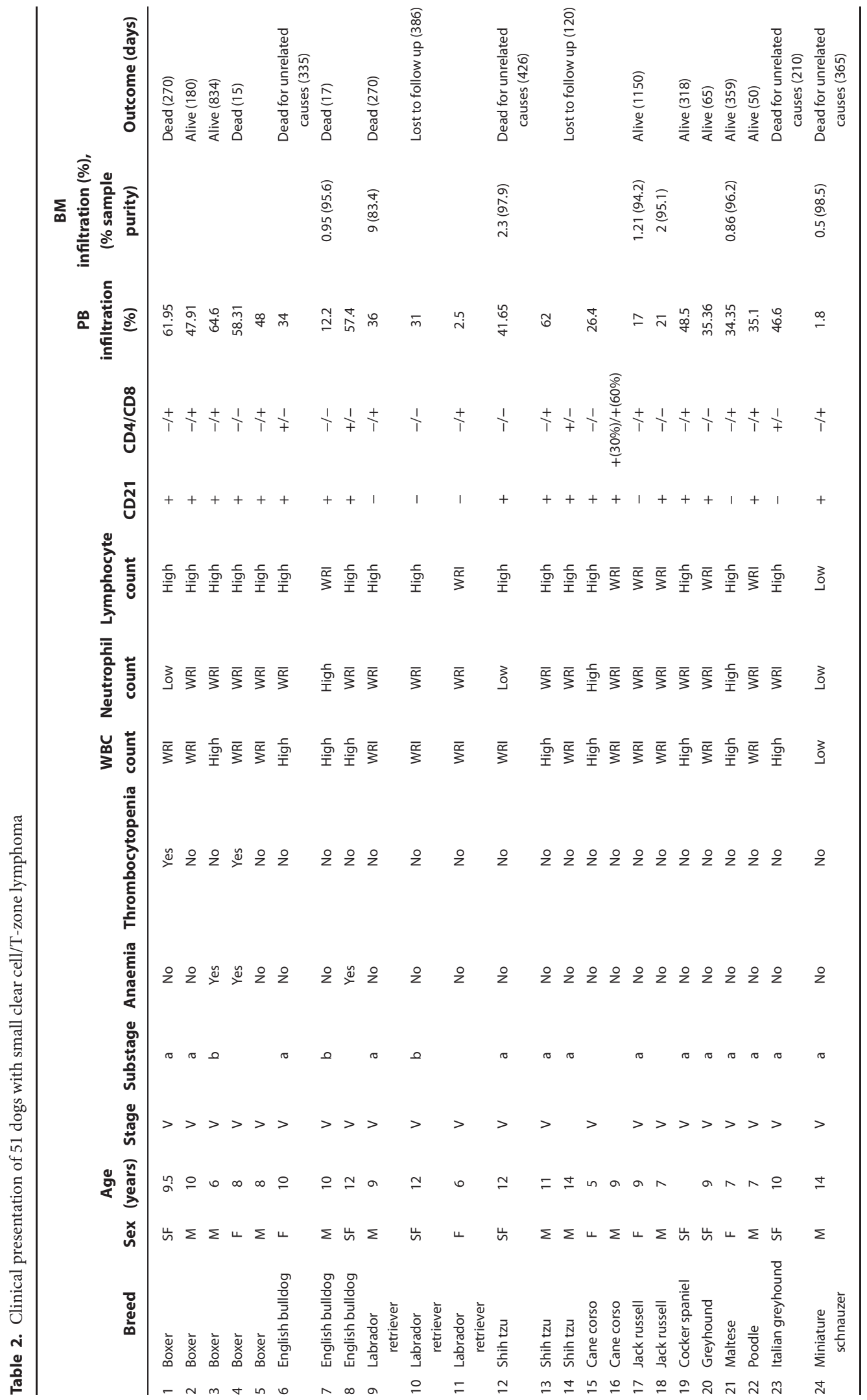




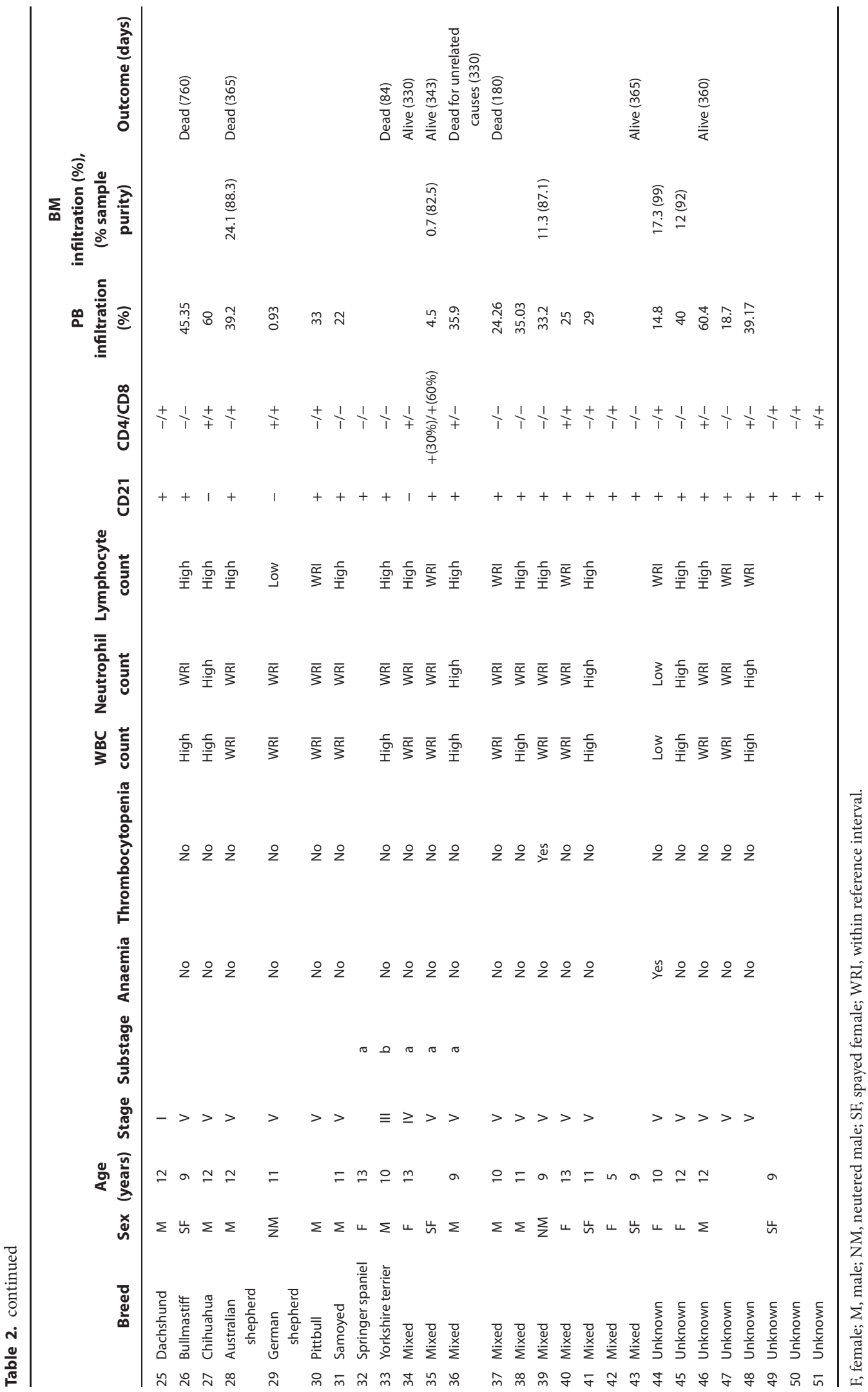


months. Among the remaining 18 dogs, 2 were lost to follow up after 120 and 386 days, respectively, 5 dogs died because of unrelated causes and 11 were still alive at data analysis closure, with a median follow-up time of 335 days (range, 50-1150). Interestingly, three dogs (12\%) developed a second malignancy during the study period (glioma, melanoma and oral carcinoma): two of them died because of the second malignancy, whereas the dog with oral carcinoma was still alive at the end of the study after 1150 days from the diagnosis.

\section{Discussion}

The existence of a CD45-negative, small clear cell/T-zone distinct lymphoma subtype showing an indolent clinical course may be presumed in dogs. ${ }^{1-5}$ This study retrospectively describes the clinical presentation and outcome of a case series of dogs affected by this particular lymphoma subtype.

Until now, only few studies have described the clinical presentation of dogs with TZL., In agreement with previous data, adult dogs are usually affected, and there seems to be no sex predilection. ${ }^{3}$ In previous reports, Golden retrievers were the dominant breed, suggesting a possible genetic risk factor. ${ }^{3}$ Interestingly, no Golden retrievers were present in our case series, possibly being attributable to a different genetic background among countries or, less likely, to a lower prevalence of this breed in Italy. Further studies should be performed to highlight possible similarities and differences in the genetics within the Golden retriever population.

Although staging was not undertaken in every dog, the majority of dogs in the present case series had an advanced clinical stage at presentation, in agreement with a previous study. ${ }^{6}$ Despite this and in contrast to high grade T-cell lymphomas, prognosis was not inexorably poor, thereby questioning the utility of the WHO system to stage indolent lymphomas. It is possible that other variables may show a more useful prognostic significance for this lymphoma subtype.

When available, PB and BM were always infiltrated. Neoplastic cell percentages were mostly higher in PB samples compared with BM samples. This phenomenon may be because of an overspill phenomenon, when nodal neoplastic cells are released into the blood circulation, rather than to BM invasion and homing. However, TZL maintains a nodular pattern in the LN, as demonstrated by histopathology. The mechanisms underlying this peculiar distribution of neoplastic cells in the organism should be investigated via further studies. A role might be played by the lack of CD45, since the inhibition of this phosphatase impaired motility and homing of both normal and leukemic humans cells in a recent study. ${ }^{11}$ The small degree of BM infiltration might also explain the low prevalence of peripheral cytopenias reported in the present study and in the study by Seelig et al. ${ }^{3}$

The median survival in this study is comparable with those already reported in the literature. ${ }^{3-5}$ Interestingly, three dogs died within a short period from the diagnosis. Two of them showed clinical symptoms at diagnosis, whereas substage was not reported for the third case. Substage b is a well-known negative prognostic factor in canine high grade lymphomas. The same prognostic significance may be held true in indolent lymphomas, thereby explaining the short survival of these dogs.

About $10 \%$ of the cases included here developed a second malignancy. This event has already been reported in $\operatorname{dogs}^{12}$ and people ${ }^{13-15}$ with lymphoma. The causes underlying the development of second malignancies are still unclear, but treatment, above all alkylating agents, has been associated with subsequent malignant neoplasms in human medicine. ${ }^{16-18}$ Two of three dogs developing second malignancy in this study had received alkylating chemotherapy.

Another possible explanation for second malignancy development is a genetic predisposition to cancer. Indeed, neoplastic transformation is based on genetic abnormalities of many different genes (oncogenes, tumour-suppressor genes and stability genes), which can occur in the germ line, resulting in hereditary predisposition to different types of cancer or in somatic cells, resulting in sporadic tumours. ${ }^{19}$

The major limits of this study are inherent to its retrospective nature. Indeed, for almost half of the included population, clinical data were missing because not reported or retrieved by the referring veterinarians. Also, staging workup varied among 
veterinarians, possibly leading to under-staging of cases. Finally, we were not able to perform survival analysis because of the huge variety of treatment regimens adopted. Unfortunately, treatment in dogs with lymphoma are not standardized yet and also the choice whether to treat or not is left to owners.

In conclusion, this study reports the clinical presentation and the outcome of a series of dogs diagnosed with CD45-negative small clear cell/TZL. The majority of dogs had stage $\mathrm{V}$ disease, were not symptomatic (substage a) and peripheral cytopenias were uncommon. The reported predisposition of Golden retrievers to develop TZL was not confirmed by our results. Canine TZL is known to bear a good prognosis with long survival times, even if a subset of dogs in our study died within few weeks. Further prospective studies on larger case series, with standardized staging workup and treatment regimens, and longer follow-up times are needed to confirm our results.

\section{Acknowledgements}

The authors thank Dr Damiano Stefanello and all the private vets who provided follow-up data for this study.

\section{References}

1. Ponce F, Marchal T, Magnol JP, Turinelli V, Ledieu D, Bonnefont C, et al. A morphological study of 608 cases of canine malignant lymphoma in France with a focus on comparative similarities between canine and human lymphoma morphology. Veterinary Pathology 2010; 47: 414-433.

2. Martini V, Poggi A, Riondato F, Gelain ME, Aresu $\mathrm{L}$ and Comazzi S. Flow-cytometric detection of phenotypic aberrancies in canine small clear cell lymphoma. Veterinary and Comparative Oncology 2013. doi:10.1111/vco.12043. [Epub ahead of print].

3. Seelig DM, Avery P, Webb T, Yoshimoto J, Bromberek J, Ehrhart EJ, et al. Canine T-zone lymphoma: unique immunophenotypic features, outcome, and population characteristics. Journal of Veterinary Internal Medicine 2014; 28: 878-886.

4. Flood-Knapik KE, Durham AC, Gregor TP, Sanchez MD, Durney ME and Sorenmo KU. Clinical, histopathological and immunohistochemical characterization of canine indolent lymphoma. Veterinary and Comparative Oncology 2013; 11: 272-286.
5. Ponce F, Magnol JP, Ledieu D, Marchal T, Turinelli $\mathrm{V}$, Chalvet-Monfray K, et al. Prognostic significance of morphological subtypes in canine malignant lymphomas during chemotherapy. The Veterinary Journal 2004; 167: 158-166.

6. Aresu L, Martini V, Rossi F, Vignoli M, Sampaolo $\mathrm{M}$, Aricò $\mathrm{A}$, et al. Canine indolent and aggressive lymphoma: clinical spectrum with histologic correlation. Veterinary and Comparative Oncology 2013. doi:10.1111/vco.12048. [Epub ahead of print].

7. Fournel-Fleury C, Ponce F, Felman P, Blavier A, Bonnefont C, Chabanne L, et al. Canine T-cell lymphomas: a morphological, immunological, and clinical study of 46 new cases. Veterinary Pathology 2002; 39: 92- 109.

8. Valli VE, Vernau W, de Lorimier LP, Graham PS and Moore PF. Canine indolent nodular lymphoma. Veterinary Pathology 2006; 43: 241-256.

9. Gelain ME, Mazzilli M, Riondato F, Marconato L and Comazzi S. Aberrant phenotypes and quantitative antigen expression in different subtypes of canine lymphoma by flow cytometry. Veterinary Immunology and Immunopathology 2008; 121: 179-188.

10. Brooimans RA, Kraan J, van Putten W, Cornelissen JJ, Löwenberg B and Gratama JW. Flow cytometric differential of leukocyte populations in normal bone marrow: influence of peripheral blood contamination. Cytometry Part B 2009; 76: 18-26.

11. Shivtiel S, Lapid K, Kalchenko V, Avigdor A, Goichberg P, Kalinkovich A, et al. CD45 regulates homing and engrafment of immature normal and leukemic human cells in transplanted immunodeficient mice. Experimental Hematology 2011; 39: 1161 - 1170 .

12. Marconato L, Stefanello D, Valenti P, Bonfanti U, Comazzi S, Roccabianca P, et al. Predictors of long-term survival in dogs with high-grade multicentric lymphoma. Journal of the American Veterinary Medical Association 2011; 238: 480-485.

13. Xu Y, Wang H, Zhou S, Yu M, Wang X, Fu K, et al. Risk of second malignant neoplasms after cyclophosphamide-based chemotherapy with or without radiation therapy for non-Hodgkin lymphoma. Leukemia \& Lymphoma 2013; 54: 1396- 1404.

14. Tajika M, Matsuo K, Ito H, Chihara D, Bhatia V, Kondo S, et al. Risk of second malignancies in patients with gastric marginal zone lymphomas of mucosa associate lymphoid tissue (MALT). Journal of Gastroenterology 2014; 49: 843-852.

15. Chowdhry AK, McHugh C, Fung C, Dhakal S, Constine LS and Milano MT. Second primary head and neck cancer after hodgkin lymphoma: a 
population-based study of 44,879 survivors of hodgkin lymphoma. Cancer 2015; 121: 1436-1445.

16. Daniels LA, Krol ADG, Schaapveld M, Putter H, Jansen PM, Marijt EWA, et al. Long-term risk of secondary skin cancers after radiation therapy for Hodgkin's lymphoma. Radiotherapy and Oncology 2013; 109: 140- 145 .

17. Ahmadzadeh A, Yekaninejad MR, Jalili MH, Bahadoram M, Efazat M, Seghatoleslami M, et al. Evaluating the survival rate and the secondary malignancies after treating hodgkin's lymphoma patients with chemotherapy regimens. International Journal of Hematology-Oncology and Stem Cell Research 2014; 8: 21-26.

18. LeMieux M, Solanki AA, Mahmood U, Chmura SJ and Koshy M. Risk of second malignancies in patients with early stage classical Hodgkin's lymphoma treated in a modern era. Cancer Medicine 2015; 4: 513-518.

19. Vogelstein B and Kinzler KW. Cancer genes and the pathways they control. Nature Medicine 2004; 10: 789-799. 